\section{Der natürliche Verlauf und die Persistenz von Halo-Nävi}

Das immer wieder erstaunliche Auftreten des weißen Hofes um einen Nävus veranlasst viele Patienten, ihren behandelnden Arzt zu fragen, wie lange dieses Phänomen andauert. Den Verlauf bei diesen Halo-Nävi, auch Sutton-Nävi genannt, untersuchten Dermatologen aus den USA.

B ei einem Halo-Nävi kommt es im Rahmen einer Immunantwort zur Störung der Melaninogenese oder zum Abbau melanozytärer Zellen durch TLymphozyten. Den spontanen Verlauf dieser Sutton-Nävi prüften Ärzte der Northeast Ohio Medical University anhand von Krankenakten niedergelassener Hautärzte aus den Jahren 19942010. Es wurden 52 Patienten mit insgesamt 80 Halo-Nävi gefunden, nachfolgend angeschrieben und $\mathrm{zu}$ einer weiteren Besprechung eingeladen. Insgesamt 56 der 80 Nävi konnten mit Hilfe eines Fragebogens und einer zusätzlichen Untersuchung dann weiter verfolgt werden (von 36 Patienten, die sich gemeldet hatten).

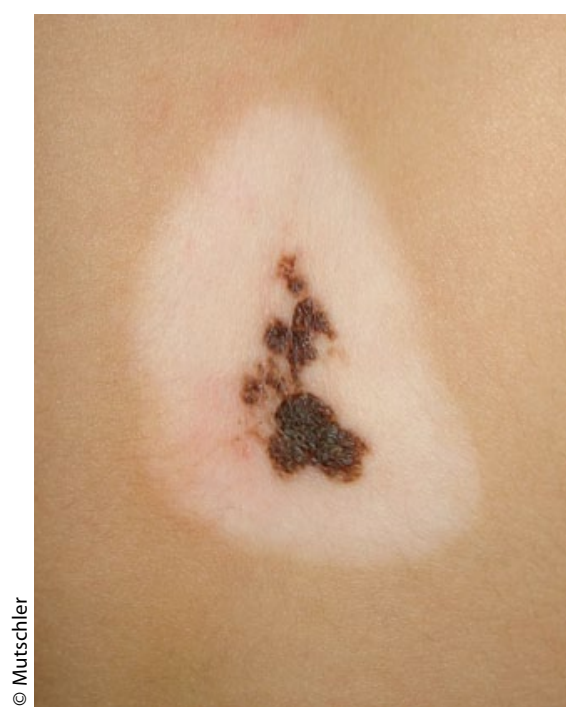

Halo-Nävi, auch Sutton-Nävi genannt

Im weiteren Verlauf wurden sieben Nävi exzidiert und histologisch untersucht, sechs aus eher kosmetischen Gründen; bei einem lag der Verdacht auf ein Melanom vor, was dann abschließend leider bestätigt werden konnte.

Von den restlichen 49 blieben 25 während eines durchschnittlichen Zeit- raums von ca. vier Jahren unverändert, sieben waren $\mathrm{z}$. T. rückläufig, aber auch nach circa $6^{1 / 2}$ Jahren war der Ring noch erhalten. Zwei Nävi waren verschwunden, aber die Depigmentierung noch sichtbar (Follow-up im Mittel knapp acht Jahre). Bei vier Nävi (8,2\%), die nach zwölf Jahren nicht mehr sichtbar waren, konnte eine teilweise Repigmentierung verzeichnet werden. Bei elf der ursprünglichen Halo-Nävi war nach durchschnittlich 7,8 Jahren weder ein Nävus noch eine Depigmentierung mehr zu sehen.

Aouthmany $\mathrm{M}$ et al. The natural history of halo Dermatol 2012; 67: 582-6

Kommentar: Sutton beschrieb 1916 erstmals ausführlich den später nach ihm benannten Befund [Journal of Cutaneous Disease Including Syphilis (Chicago) 1916; 34 : 797-800]. Es handelt sich dabei um melanozytäre Nävi mit unpigmentiertem Hof (Halo), meist als Zufallsbefund bei Personen mit multiplen dunklen Naevi, gehäuft bei Patienten mit Vitiligo oder auch bei metastasierenden Melanomen. Halo-Nävi kommen oft bei Jugendlichen oder jungen Erwachsenen und dann häufig am Stamm, vor allem am Rücken, vor. Eine Exzision sollte bei klinischem Malignitätsverdacht nach der sog. $A B C D$-Regel erfolgen. Bisher war wenig über den weiteren Verlauf bekannt: Sieht man sich nun die Zahlen aus der vorliegenden Arbeit an, können Sutton-Nävi sehr lange - also zehn Jahre und länger - bestehen. Eine Untergruppe von etwa einem Fünftel verschwindet nach etwa acht Jahren. Um falsche Erwartungen, aber auch Ängste zu vermeiden, sollte mit den Patienten ausführlich darüber gesprochen werden. Gleichzeitig sind regelmäßige Followups indiziert, um Fälle wie das oben erwähnte Melanom rechtzeitig zu entdecken. Dr. Ulrich Mutschler nevi: a retrospective case series. J Am Acad

Springer Springer Springer Springer $\underline{\text { Springer }}$ Springe Springer $\underline{\text { Springer }}$ Springer Springer Springer $\underline{\text { Springe }}$ Q 2 Springer $\underline{\text { Springer }}$ Springer Q Springer $\underline{\text { Springer }}$ Springe Springer 2 Springer 2 Springer Q Springer $\underline{\text { Springer }}$ Springe Q 2 Springer $\underline{\text { Springer }}$ Springer

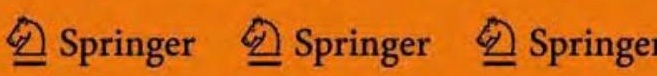

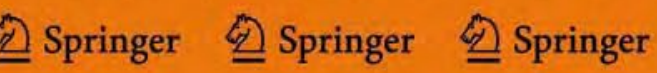

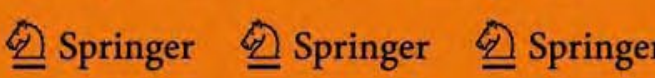
은 Springer $\underline{\text { Springer }}$ Springer $\underline{\text { Springer }}$ Springer $\underline{\text { Springer }}$ Springer $\triangleq$ Springer $\cong$ Springer Q Springer $\underline{\text { Springer }}$ Springer Springer $\triangleq$ Springer $\underline{\text { Springer }}$ Springer $\underline{\underline{S} \text { Springe }}$

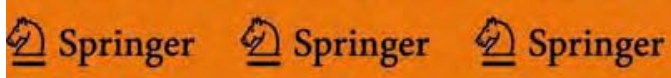
Q Springer $\underline{\text { Springer }}$ Springe QSpringer $\underline{\text { Springer }}$ Springer Springer Springer 2 Springer 은 Springer $\underline{\text { Springer }}$ Springer Springer $\underline{Q}$ Springer $\underline{\text { Springe }}$ Q Springer Springer 2 Springer 은 Springer Springer Springer Springer $\underline{\text { Springer }}$ Springer Q Springer $\underline{\underline{S} \text { Springer }}$ Springe

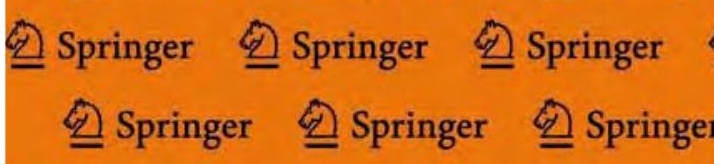
Q Springer 2 Springer Springer Springer $\underline{\text { Springer }}$ Springe Springer $\underline{\underline{2} \text { Springer }}$ Springer 